\title{
Temperature Dependent Tuning of Defect Mode inside Photonic Bandgap for Cwdm Applications
}

\author{
Ranjita Panda $^{1}$, Maitreyi Upadhyay ${ }^{2}$, Suneet Kumar Awasthi ${ }^{2, *}$ \\ ${ }^{1}$ Department of Physics, School of Basic Science and Research, Sharda University, Uttar Pradesh, India \\ ${ }^{2}$ Department of Physics and Material Science and Engineering, Jaypee Institute of Information Technology, Noida, India
}

\section{Email address:}

suneet_electronic@yahoo.com (S. K. Awasthi)

${ }^{*}$ Corresponding author

\section{To cite this article:}

Ranjita Panda, Maitreyi Upadhyay, Suneet Kumar Awasthi. Temperature Dependent Tuning of Defect Mode inside Photonic Bandgap for Cwdm Applications. Optics. Vol. 6, No. 1, 2017, pp. 5-10. doi: 10.11648/j.optics.20170601.12

Received: June 30, 2017; Accepted: July 10, 2017; Published: August 11, 2017

\begin{abstract}
A theoretical study of temperature dependent tuning of defect mode inside photonic bandgap of one-dimensional photonic crystal with binary defect for optical communication applications based on coarse wavelength division multiplexing (CWDM) is presented. The transmission properties of the proposed structure are investigated through the transfer matrix method (TMM) at normal incidence. The proposed contraption is capable of selecting and switching any one wavelength channel at a time centered at particular wavelength out of $12 \mathrm{CWDM}$ channels as per the international telecommunication union (ITU) grid G.694.2. It is very useful for narrowband wavelength selective switching (NWSS) applications based on CWDM. This study also provides some insight to design new kind of thermally tunable optical filters which can be used effectively in remote sensing and thermal imaging applications.
\end{abstract}

Keywords: Photonic Band-Gap Materials, Optical Switching, Optical Filters

\section{Introduction}

Photonic crystals (PCs) have been the object of intensive studies in the past two and half decades due to their unique electromagnetic properties and potential applications [1-10]. PCs are the periodic structures in which refractive index changes periodically in space. Such structure affects the propagation of electromagnetic wave in similar way that the periodic potential in semiconductor crystal affects electron motion. Introduction of extra element into PC destroys the periodicity and leads to the appearance of the narrow peaks with high transmittance at a frequency inside the PBG, socalled defect modes. This property of one-dimensional (1D) defect photonic crystal (DPC) is very useful for designing of tunable optical filters which have potential applications in wavelength division multiplexing (WDM) based optical networks because of their ability to control propagation of light. Analytical and numerical calculations of the defect mode tunneling through 1D DPCs have been carried out by many authors [11-15] but none of them extended their work to study thermally tunable narrowband wavelength selective switching (NWSS) properties of 1D DPC for optical communication applications based on CWDM.

In this work, temperature dependent tuning of defect mode inside PBG for CWDM applications is presented. The proposed structure can select and switch only one wavelength channel at a time centered at one particular wavelength channel amongst $12 \mathrm{CWDM}$ channels as per the ITU grid G.694.2 [16]. Though the spectral width of each channel of conventional CWDM channels should be between 13-15 nm, the spectral width of the defect mode of the proposed filter is much smaller of the order $4 \mathrm{~nm}$ and thus makes the structure to be used very effectively for NWSS applications. It is also capable of becoming a key component of WDM based optical networks for enlarging their capacity and flexibility by overcoming the inherent limitations of a semiconductor optical amplifier (SOA) based wavelength selective switches, such as power consumption, signal to noise ratio, speed, polarization and size etc. [17-18]. The key idea of the proposed filter is based on the temperature variation between $295 \mathrm{~K}$ to $1522 \mathrm{~K}$ for switching number of CWDM channels one by one at a time.

The structure of manuscript is organized as follows: The 
theoretical formulation is introduced in Section 2. Numerical results are presented and discussed in Section 3. Finally, conclusions are given in Section 4.

\section{Theoretical Formulation}

We consider two finite size two-component dielectric PCs of structure $(\mathrm{AB})^{N}$ placed together to form the structure
$(\mathrm{AB})^{N} \mathrm{DB}(\mathrm{AB})^{N}$ with two defect layers, as shown in Figure 1. Here $A$ and $B$ stand for the different dielectric layers with high and low refractive indices $n_{A}$ and $n_{B}$ respectively, $D$ is the defect layer and $\mathrm{N}$ is period number. The thicknesses of material layer $\mathrm{A}$ and $\mathrm{B}$ are denoted by $d_{\mathrm{A}}$ and $d_{\mathrm{B}}$. The refractive index and thickness of the defect layer $\mathrm{D}$ is represented by $n_{\mathrm{d}}$ and $d_{\mathrm{d}}$ respectively.

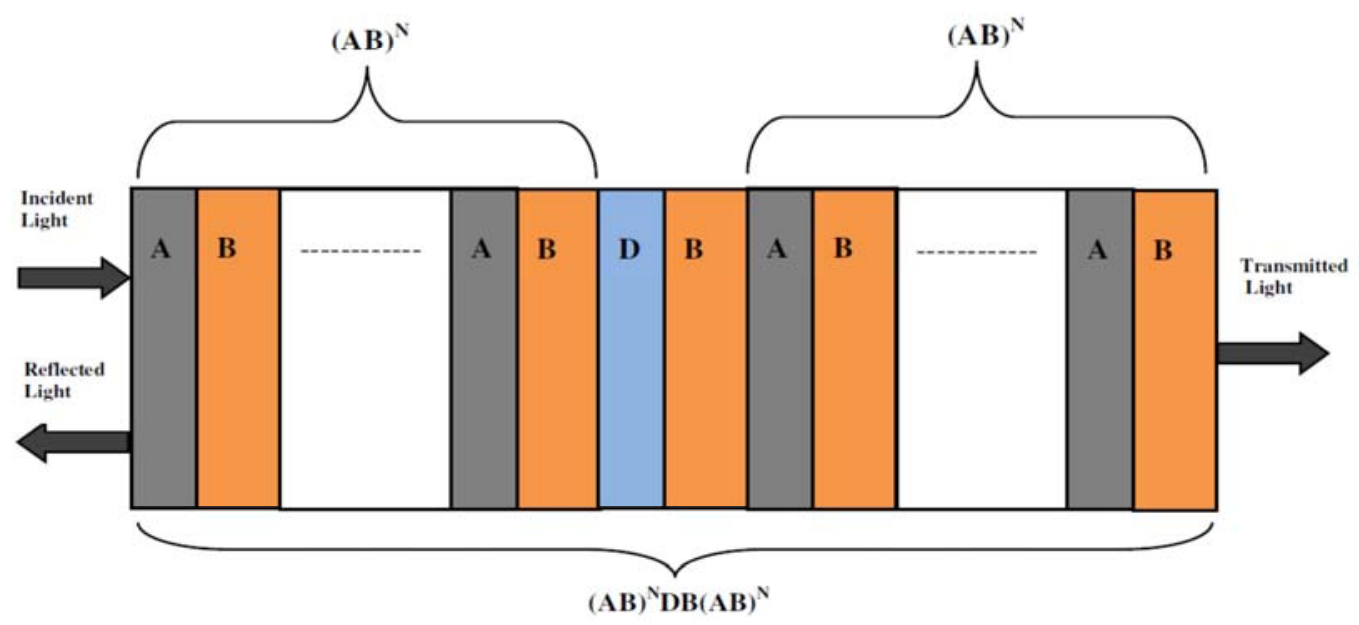

Figure 1. Systematic design of $1 D D P C$.

In order to obtain transmission spectra of proposed 1D DPC we have chosen TMM, which is the most effective way to analyze the transmission properties of periodic and nonperiodic multilayer structures [12-14]. The characteristic matrix $M(\Lambda)$ of one period of any PC composed of two material layers is given by $[9,14,19]$

$$
M(\Lambda)=\left(\begin{array}{ll}
M_{11} & M_{12} \\
M_{21} & M_{22}
\end{array}\right)=\prod_{K=1}^{2}\left(\begin{array}{cc}
\cos \delta_{k} & -\frac{i}{q_{k}} \sin \delta_{k} \\
-i q_{k} \sin \delta_{k} & \cos \delta_{k}
\end{array}\right)
$$

Where $q_{k}=n_{k} \cos \theta_{k},(\mathrm{k}=1,2$ represent layers $\mathrm{A}$ and $\mathrm{B}$ respectively) and $\delta_{k}=\frac{2 \pi}{\lambda_{0}} n_{k} d_{k} \cos \theta_{k}$ here $\theta_{k}$ is the ray angle inside the layer of refractive index $n_{\mathrm{k}}$ with thickness $d_{\mathrm{k}}$ and $\lambda_{0}$ is the free space wavelength. The characteristic matrix representing $\mathrm{N}$ periods of same $\mathrm{PC}$ is given by,

$$
M(N \Lambda)=[M(\Lambda)]^{N}=\left(\begin{array}{ll}
M_{11} & M_{12} \\
M_{21} & M_{22}
\end{array}\right)^{N}
$$

Now the characteristic matrix representing left and right PCs which are on either side of binary defect layers (DB) and is given by [12]

$$
M_{L}^{P C}=\left(M_{1} M_{2}\right)^{N} \text { and } M_{R}^{P C}=\left(M_{1} M_{2}\right)^{N}
$$

The characteristic matrix of the binary defect layers DB is of the form
$M_{d}=\left(\begin{array}{cc}\cos \delta_{d} & -\frac{i}{q_{d}} \sin \delta_{d} \\ -i q_{d} \sin \delta_{d} & \cos \delta_{d}\end{array}\right)\left(\begin{array}{cc}\cos \delta_{b} & -\frac{i}{q_{b}} \sin \delta_{b} \\ -i q_{b} \sin \delta_{b} & \cos \delta_{b}\end{array}\right)$

where $q_{d}=n_{d} d_{d} \cos \theta_{d}, \delta_{d}=\frac{2 \pi}{\lambda_{0}} n_{d} d_{d} \cos \theta_{d}$,

$q_{b}=n_{b} d_{b} \cos \theta_{b}$ and $\delta_{b}=\frac{2 \pi}{\lambda_{0}} n_{b} d_{b} \cos \theta_{b}$

Here $\theta_{\mathrm{d}}$ and $\theta_{\mathrm{b}}$ are the ray angle inside defect layer D and $\mathrm{B}$ respectively. The total characteristic matrix representing 1D DPC can be expressed as

$$
M_{T}=M_{L}^{P C} M_{d} M_{R}^{P C}=\left(\begin{array}{ll}
m_{11} & m_{12} \\
m_{21} & m_{22}
\end{array}\right)
$$

The transmission coefficient of 1D DPC is obtained to be

$$
t=\frac{2 q_{0}}{\left(m_{11}+m_{12} q_{s}\right) q_{0}+\left(m_{21}+m_{22} q_{s}\right)}
$$

The values of $q_{0}$ and $q_{\mathrm{s}}$ used in equation (6) are given as $q_{0}=n_{0} \cos \theta_{0}$ and $q_{s}=n_{s} \cos \theta_{s}$. Here $\theta_{0}$ is the incident angle and $\theta_{s}$ represents ray angle inside substrate. The transmittance of 1D DPC is given by [9]

$$
T=|t|^{2}=\left(t \times t^{*}\right)
$$


The wavelength and temperature dependent refractive index of silicon $(\mathrm{Si})$ in the ranges 1.2 to $14 \mu \mathrm{m}$ and 293-1600 $\mathrm{K}$ respectively is defined as [17]

$$
\mathrm{n}(\lambda, \mathrm{T})=\sqrt{\varepsilon(\mathrm{T})+\frac{\mathrm{L}(\mathrm{T})}{\lambda^{2}}\left(\mathrm{~A}_{0}+\mathrm{A}_{1} \mathrm{~T}+\mathrm{A}_{2} \mathrm{~T}\right)}
$$

Where

$$
\begin{gathered}
\mathcal{E}(T)=11.4445+2.7739 \times 10^{-4} T+1.7050 \times 10^{-6} T^{2}-8.1347 \times 10^{-10} T^{3} \\
\mathrm{~L}(\mathrm{~T})=\mathrm{e}^{-3 \Delta \mathrm{L}(\mathrm{T}) / \mathrm{L}_{293}}
\end{gathered}
$$

$\lambda=$ wavelength in units of $\mu \mathrm{m}$,

$T=$ temperature in units of $\mathrm{K}$,

$$
A_{o}=0.8948, A_{1}=4.3977 \times 10^{-4}, A_{2}=7.3835 \times 10^{-8},
$$

and

$$
\frac{\Delta L(T)}{L_{293}}=0.071+1.887 \times 10^{-6} T+1.934 \times 10^{-9} T^{2}-4.544 \times 10^{-13} T^{3}
$$
by

The temperature dependent thickness of Si layer is given

$$
d(T)=d_{293}(1+\alpha \Delta T)=d_{293}\left[1+\frac{\Delta L(T)}{L_{293}}\right]
$$

Where $d_{293}$ and $d(T)$ represent the thicknesses of Si layer at temperatures $293 \mathrm{~K}$ and $T \mathrm{~K}$ respectively, $\alpha$ is the coefficient of thermal expansion of $\mathrm{Si}$ with numerical value $2.5 \times 10^{-6} / \mathrm{K}$ and $\Delta T$ is the change in temperature of $\mathrm{Si}$ layer. Due to the thermal expansion in $\mathrm{Si}$ layer, the thicknesses $d_{\mathrm{A}}$ and $d_{\mathrm{B}}$ of layers $\mathrm{A}$ and $\mathrm{B}$ of $\mathrm{PC}$ at temperature $293 \mathrm{~K}$ will be modified in accordance with Eq. (10) as

$$
d_{A}(T)=d_{A}+\Delta d_{A} \text { and } d_{B}(T)=d_{B}+\Delta d_{B}
$$

Here $\Delta d_{i}=d(T)-d_{i}(293)$ is the change in the thickness of $i^{\text {th }}$ layer due to corresponding change in temperature $\Delta \mathrm{T}$. The subscript $i=\mathrm{A}$ and $\mathrm{B}$ are used to represent $\mathrm{Si}$ and air layers respectively.

\section{Results and Discussion}

In this section, narrowband temperature dependent transmission characteristics of defect mode inside PBG for switching optical communication channels used in CWDM based optical networks is presented. In order to obtain transmission spectra the TMM is used at normal incidence. We have chosen dielectric materials $\mathrm{Si}$ and air to represent layers $\mathrm{A}$ and $\mathrm{B}$ of the proposed structure respectively. The refractive index of air is 1 . The thicknesses of layers $A$ and $B$ are $d_{\mathrm{A}}=106.1 \mathrm{~nm}$ and $d_{\mathrm{B}}=181.25 \mathrm{~nm}$ in order to get wider $\mathrm{PBG}$ in the near-infrared spectral region, because this is a low-loss region in silica fibers [18]. The working principle of the proposed structure works on the temperature dependent tuning of the defect mode inside PBG in such a way that any change in temperature of DPC changes the thicknesses of layers A and B according with Eq. (10) which in turn influences the range of $\mathrm{PBG}$ of $\mathrm{PC}$. The transmission spectra at normal incidence of $\mathrm{PC}(\mathrm{AB})^{5}$ without defect at temperature $293 \mathrm{~K}$ is plotted as a function of wavelength in Figure 2. It shows that the PBG extending from $\lambda_{l}=1200 \mathrm{~nm}$ to $\lambda_{r}=1623 \mathrm{~nm}$ which lies within the spectral band (1260$1675 \mathrm{~nm}$ ) used for optical fiber communications [15-16]. The PBG range of $(A B)^{5}$ is also dependent on the temperature of $\mathrm{Si}$ besides other parameters such as lattice constant, refractive indices of materials, etc.

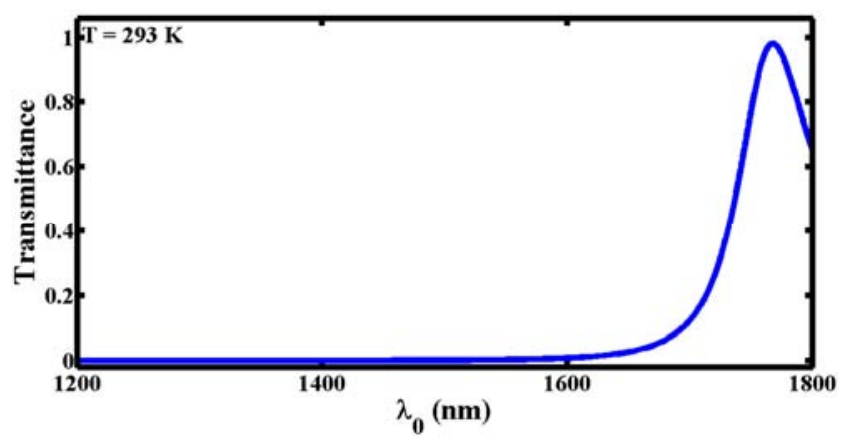

Figure 2. Transmission spectra at normal incidence of $1 D P C(A B)^{5 v}$ by blue color $\left(d_{1}=106.1 \mathrm{~nm}, d_{2}=181.25 \mathrm{~nm}, N=12\right.$ and $\left.T=293 \mathrm{~K}\right)$.

Now, we disturb the periodicity of the periodic structure $(\mathrm{AB})^{5}$ by introducing a binary defect layers $\mathrm{D}$ and $\mathrm{B}$ which modify the structure $(\mathrm{AB})^{2} \mathrm{DB}(\mathrm{AB})^{2}$ as shown in Figure 1. The thickness of silicon defect layer is optimized as $469 \mathrm{~nm}$ to get sharp defect mode of spectral width $4 \mathrm{~nm}$ centered at wavelength $1371 \mathrm{~nm}$ inside PBG ranging from 1250 to $1650 \mathrm{~nm}$ at temperature $295 \mathrm{~K}$ while the thickness of the defect layer $\mathrm{B}$ is remains same. Figure 3 illustrates how central wavelength of defect mode can be modulated from $1371 \mathrm{~nm}$ to $1591 \mathrm{~nm}$ in intervals of $20 \mathrm{~nm}$ by precisely adjusting the temperature of $\mathrm{Si}$ defect layer. This temperature dependent tunable behavior of defect mode is used to select and switch desired wavelength channel at a time from 12 CWDM channels of spacing $20 \mathrm{~nm}$ as per ITU-T G.694.2 (Table 1). These results also demonstrate that the temperature dependent transmittance of each CWDM channel is almost higher than 99\% (-0.1dB) which is very essential because it causes very low insertion loss [20]. This parameter is very useful for power budget calculation and network planning.

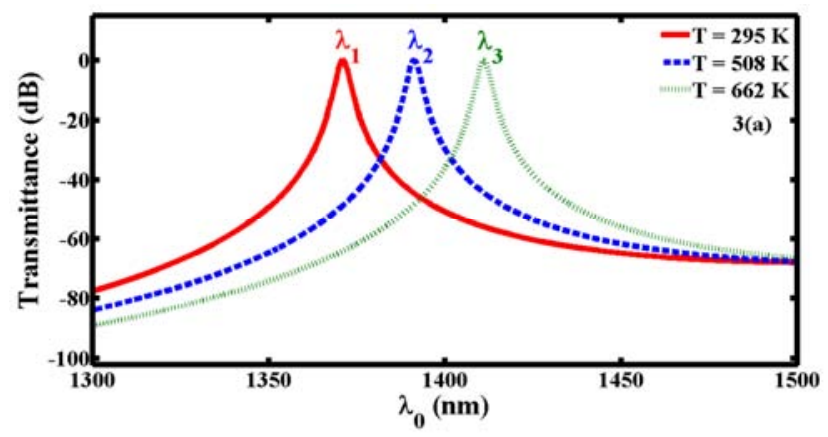



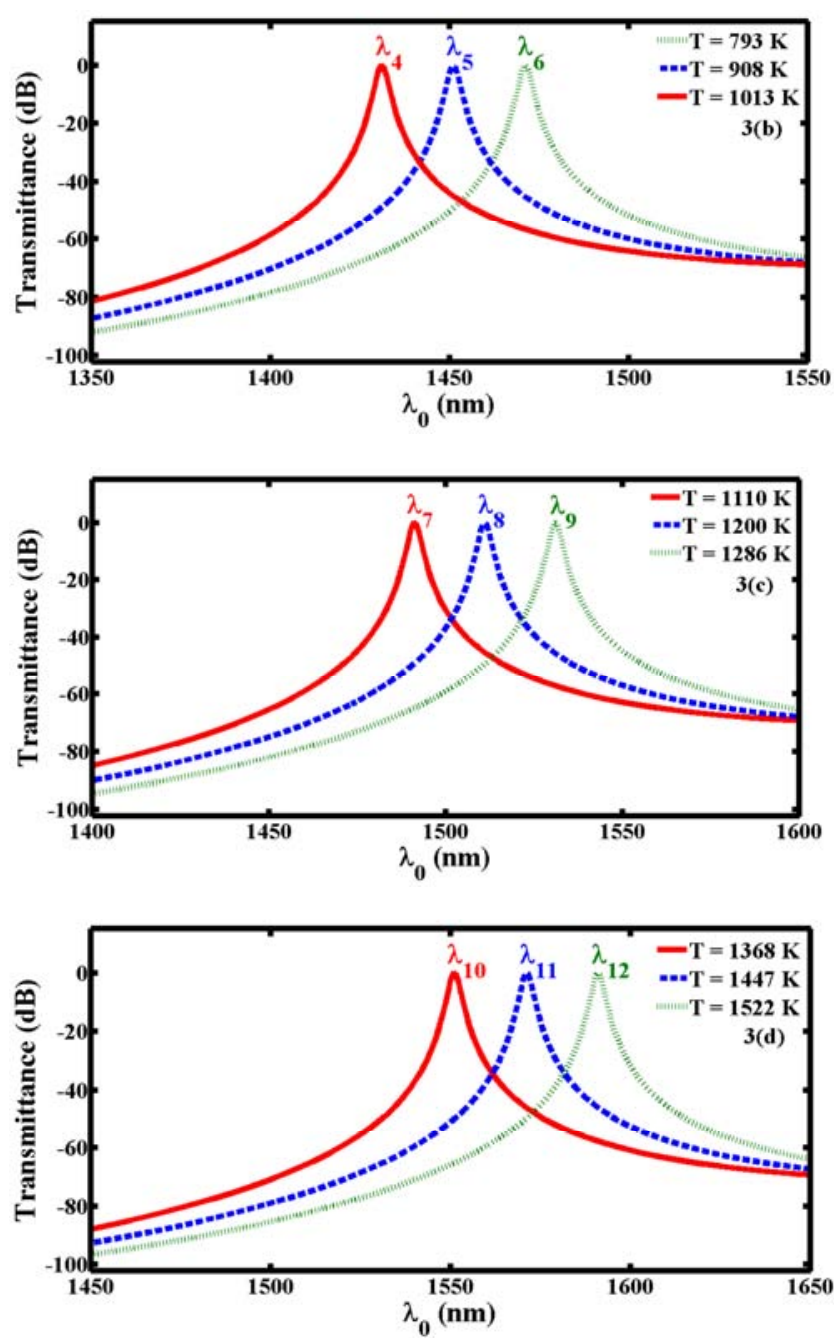

Figure 3. (a)-(d). Transmission spectra of single channel thermally tunable optical filter featuring the defect mode inside PBG of $1 D$ DPC at different temperatures.
Table 1. ITU-Based 12 CWDM channels with their separation.

\begin{tabular}{llll}
\hline S. No. & CWDM Channel & $\begin{array}{l}\text { Center } \\
\text { Wavelength (nm) }\end{array}$ & $\begin{array}{l}\text { Channel } \\
\text { Separation (nm) }\end{array}$ \\
\hline 1. & $\lambda_{1}$ & 1371 & 20 \\
2. & $\lambda_{2}$ & 1391 & 20 \\
3. & $\lambda_{3}$ & 1411 & 20 \\
4. & $\lambda_{4}$ & 1431 & 20 \\
5. & $\lambda_{5}$ & 1451 & 20 \\
6. & $\lambda_{6}$ & 1471 & 20 \\
7. & $\lambda_{7}$ & 1491 & 20 \\
8. & $\lambda_{8}$ & 1511 & 20 \\
9. & $\lambda_{9}$ & 1531 & 20 \\
10. & $\lambda_{10}$ & 1551 & 20 \\
11. & $\lambda_{11}$ & 1571 & 20 \\
12. & $\lambda_{12}$ & 1591 & 20 \\
\hline
\end{tabular}

Moreover the spectral width $(\Delta \lambda$ is the wavelength range around ITU central wavelength) of each CWDM channel starts to increase as the central wavelength of the channel under consideration shift towards higher wavelength side depending upon various values of temperature in order to compensate the temperature effects. All these observations are summarized in Table 2. It is also clearly evident from Table 2 that at fixed spectral width the frequency band occupied by the temperature dependent defect mode (called CWDM channel) decreases which results decrease in $3 \mathrm{~dB}$ optical bandwidth of defect mode towards higher wavelength side as given in Table 3 (lower frequency side). This study may find very useful applications to upgraded CWDM systems in which individual channels of the original system are replaced with sub-bands of more densely packed upgraded channels to deliver more services over the existing optical fiber infrastructure by combining multiple wavelengths on a single fiber. It can also be helpful in designing some new kind of tunable optical devices for measuring temperature precisely to ensure their potential applications in remote sensing and thermal imaging.

Table 2. Control table showing temperature dependent behavior of defect mode.

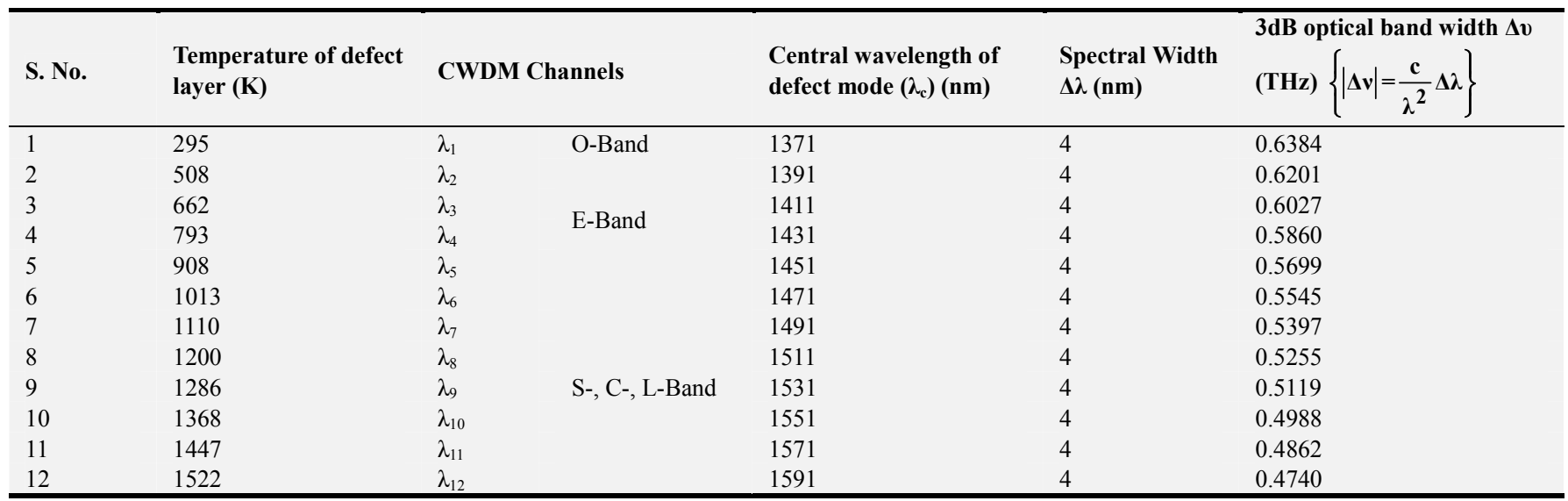

Furthermore Figure 3 shows that the defect mode of the proposed filter is very sensitive to the structure temperature and has a sharp slope, no side lobes (which results almost negligible cross talk), low insertion loss, standard spectral width $(\Delta \lambda=4$ $\mathrm{nm}$ at $3 \mathrm{~dB})$. Thus it makes the contraption to be used effectively for NWSS applications. Since maximum spectral width of the defect mode at any value of temperature is $4 \mathrm{~nm}$, which is much below the spectral width reported in [20]. Therefore the structure proposed by us can be a better choice for narrowband filtering applications in CWDM based optical networks. 
Table 3. Central wavelengths of defect modes and their corresponding crosstalk's.

\begin{tabular}{|c|c|c|c|c|c|}
\hline \multirow[b]{2}{*}{ S. No. } & \multirow{2}{*}{$\begin{array}{l}\text { Central wavelength of } \\
\text { defect mode }\left(\lambda_{c}\right)(\mathrm{nm})\end{array}$} & \multicolumn{4}{|c|}{ Percentage transmittance of adjacent defect mode and corresponding Crosstalk } \\
\hline & & On the longer wavelength side of $\lambda_{\mathrm{c}}$ & Crosstalk (dB) & $\begin{array}{l}\text { On the shorter wavelength side } \\
\text { of } \lambda_{c}\end{array}$ & Crosstalk (dB) \\
\hline 1 & 1371 & 0.0028 & -45.52 & 0.0022 & -46.57 \\
\hline 2 & 1391 & 0.0035 & -44.55 & 0.0023 & -46.38 \\
\hline 3 & 1411 & 0.0039 & -44.08 & 0.0028 & -45.52 \\
\hline 4 & 1431 & 0.0047 & -43.27 & 0.0033 & -44.81 \\
\hline 5 & 1451 & 0.0060 & -42.21 & 0.0037 & -44.31 \\
\hline 6 & 1471 & 0.0073 & -41.30 & 0.0044 & -43.56 \\
\hline 7 & 1491 & 0.0091 & -40.40 & 0.0053 & -42.75 \\
\hline 8 & 1511 & 0.0112 & -39.50 & 0.0066 & -41.80 \\
\hline 9 & 1531 & 0.0147 & -38.32 & 0.0078 & -41.07 \\
\hline 10 & 1551 & 0.0181 & -37.39 & 0.0099 & -40.04 \\
\hline 11 & 1571 & 0.0242 & -36.17 & 0.0119 & -39.24 \\
\hline 12 & 1591 & 0.0293 & -35.33 & 0.0159 & -37.98 \\
\hline
\end{tabular}

Figure 4 depicts the variation of central wavelength of the defect mode with the temperature and determines the thermal stability which describes the amount of thermal drift in the central wavelength of the defect mode. Its value is $0.97 \mathrm{~nm} / \mathrm{K}$ approximately and not critical for CWDM networks [16]. Besides this the cross talk as defined in [21] between two adjacent channels (defect modes) of the proposed filter is negligible and hence improves the system performance. The cross talk between two adjacent defect modes under consideration and their central wavelength is listed in Table 3 which is much below the permissible limit of $-25 \mathrm{~dB}$.

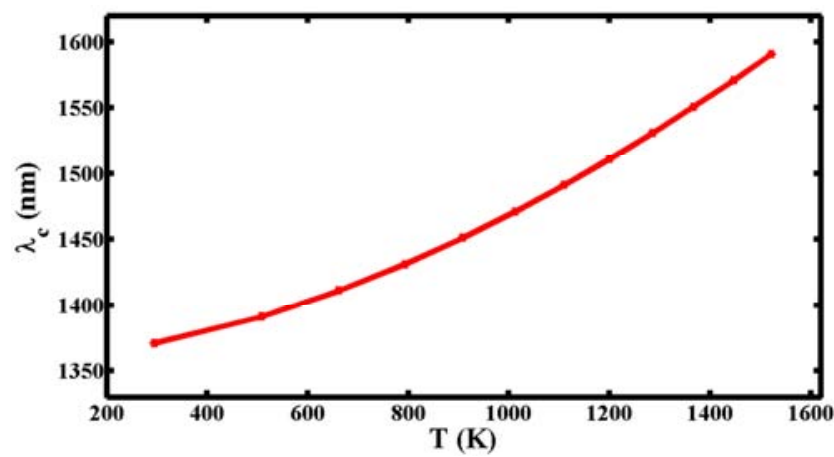

Figure 4. Temperature dependent positioning of central wavelength of defect mode to cover all 12 CWDM channels.

Moreover the comparison of the proposed filter with mod 4 down binary number sequence non periodic multilayer structure as reported in reference [22], shows that central wavelength of all $12 \mathrm{CWDM}$ channels with spectral width 4 $\mathrm{nm}$ can be filtered one by one from the proposed structure only by precisely varying the temperature of the defect layer externally whereas in order to filter all these CWDM channels from mod 4 down binary number sequence non periodic multilayer structure, we have a requirement of 12 structures with slightly different thickness of low index layer which increases the system cost and limits the use of such filters for NWSS applications. Due to this reason the proposed structure can better serve the purpose. Though the total thickness of the proposed structure varies between $29.29 \%$ to $39.17 \%$ in comparison with the structure reported in reference [22] when designed for $1591 \mathrm{~nm}$ to $1351 \mathrm{~nm}$ respectively. This enhancement in the size of the proposed structure can be ignored in comparison with the temperature dependent tuning properties the defect mode for optical communication applications based on CWDM.

\section{Conclusions}

In short the temperature dependent tuning of defect mode inside photonic bandgap of 1D DPC composed of silicon has been theoretically investigated in this work. The proposed photonic structure is capable to filter only one wavelength channel at a time centered at particular wavelength channel amongst 12 CWDM channels as per the ITU grid G.694.2. The temperature dependent defect mode of spectral width 4 $\mathrm{nm}$ has a sharp slope, no side lobes and low insertion loss and thus can be used to work efficiently for NWSS applications. This study may also be explored in designing some new kind of thermally tunable optical devices for measuring temperature precisely. Such filters may have potential applications in remote sensing and thermal imaging.

\section{Acknowledgements}

The authors would like to thank Retd. Prof. and Head Department of Physics Prof. U. Malaviya of University of Lucknow for their useful discussions and support during work.

\section{References}

[1] J. Joannopoulos, R. Meade, and J. Winn, Photonic Crystals (Princeton University, 1995).

[2] B. Glance, IEEE Photon. Technol. Letts 8, 245 (1996).

[3] J. D. Jaonnopoulos, P. R. Villeneuve, S. Fan, Nature 386, 143 (1997).

[4] K. Sakoda, Optical Properties of Photonic Crystals (Springer 2001).

[5] Y. J. Jen, A. Lakhtakia, M. J. Lin, W. H. Wang, H. M. Wu, H. S. Liao, Scientific Reports 3, 1672 (2013). 
[6] A. Mishra, S. K. Awasthi, S. K. Srivastava, U. Malaviya, S. P. Ojha, JOSA B 28, 1416 (2011).

[7] B. Suthar, A. Bhargava, IEEE Photonics Technol. Letts. 24 338 (2012).

[8] R. Ghosh, K. K. Ghosh, R. Chakraborty, Opts. Commun. 289, 75 (2013).

[9] S. K. Awasthi, U. Malaviya, S. P. Ojha, JOSA B 23, 2566 (2006).

[10] Maitreyi Upadhyay, S. K. Awasthi, Laxmi Shiveshwari, P. K. Srivastava, S. P. Ojha, J. Supercond. Nov. Magn. 28, 2275 (2015).

[11] M. Upadhyay, S. K. Awasthi, S. N. Shukla, P. Singh, $2^{\text {nd }}$ IEM OPTRONIX, IEEE, (2015).

[12] X. K. Kong, S. B. Liu, H. F. Zhang, C. Z. Li, Phys. Plasmas 17, 1035061 (2010).

[13] M. J. Steel, M. Levy and R. M. Osgood, IEEE Photon. Technol. Lett. 12, 1171 (2000).

[14] S. J. Orfanidis, "Electromagnetic waves and antennas" (Rutgers University, 2008).
[15] R. Bandyopadhyay, R. Chakraborty, Optical Engineering 54 (11), 1171105 (2015).

[16] H. J. Theile, M. Nebeling, Coarse wavelength division multiplexing technologies and applications. CRC Press, London, (2007).

[17] A. Sharkawy, S. Shi, D. W. Prather, Appl. Opt. 40, 2247 (2001).

[18] https://www.researchgate.net/publication/229005117_REALI ZATION_OF_OPTICAL_LOGIC_GATES_USING_PHOTO NIC_CRȲSTĀL

[19] M. Born, E. Wolf, Principles of Optics, pp. 1-70, (1980), United Kingdom: Cambridge University Press.

[20] Z. Y. Xiao, Z. H. Wang, Int. J. of Infrared and Millimeter Waves 25, 1315 (2004).

[21] A. Kumar, B. Suthar, V. Kumar, Kh. S. Singh, A. Bhargava, PIER Letts. 33, 27 (2012).

[22] A. Banerjee, JOSA B 26, 537 (2009). 